\section{IDDF2019-ABS-0157 FECAL MICROBIOTA TRANSPLANTATIONS RECONSTITUTE GUT FUNGAL AND VIRAL MICROBIOTA IN GRAFT-VERSUS-HOST DISEASE}

${ }^{1}$ Fen Zhang ${ }^{*}$, ${ }^{1}$ Yun Kit Yeoh, ${ }^{1}$ Tao Zuo, ${ }^{2}$ Frankie Cheng, 'Whitney Tang, ${ }^{1}$ Kitty Cheung, ${ }^{1}$ Keli Yang, ${ }^{1}$ Qin Liu, ${ }^{1}$ Chun Pan Cheung, ${ }^{2}$ Chow Chung Mo, ${ }^{1}$ Mamie Hui, ${ }^{1}$ Francis Chan, ${ }^{1}$ Chi-Kong Li, ${ }^{2}$ Paul Chan. ${ }^{1}$ Center for Gut Microbiota Research, The Chinese University of Hong Kong, Hong Kong; ${ }^{2}$ Department of Pediatrics, The Chinese University of Hong Kong, Hong Kong

\subsection{6/gutjnl-2019-IDDFabstracts.172}

Background Fecal microbiota transplant (FMT) has emerged as a potential treatment for severe colitis associated with graftversus-host disease (GvHD) following hematopoietic stem cell transplant. Bacteria engraftment has been reported, however, the fate of fungi and viruses and their relationship with treatment response after FMT remains unclear. Here we report for the first time longitudinal dynamics of the gut mycobiome and virome in a teenager with gut GvHD successfully treated with multiple FMTs.

Methods A 14-year-old boy with severe life-threatening grade4 gut aGVHD, refractory to corticosteroids and biologic therapies, were treated with a total of four FMTs. FMTs were conducted by duodenojejunal infusion from two different donors. Fecal samples were collected at baseline and up to 120 days after FMT. Combined DNA extraction of fecal bacteria and fungi was performed, followed by ultra-deep metagenomics sequencing and profiling of bacteriome and fungome. Fecal virus-like particles were enriched from feces and followed by metagenomics sequencing on VLP DNA as well as profiling of virome. Clinical phenotype and outcome were assessed and correlated with microbial profiles.

Results FMT altered the gut bacterial, fungal and viral communities simultaneously in the GvHD patient, and resulted in recovery of the patient. Bacterial diversity was gradually restored after each FMT, engraftment of donor-derived fungi occurred instantly after a single FMT and persisted up to 4 months, whilst viral diversity was improved after multiple FMTs but the composition varied substantially over time.

Moreover, FMT reduced an overrepresented fungus Fusarium oxysporum $(61.4 \%$ at day 0 vs $0.5 \%$ at day 120$)$ and virus Torque teno virus $(98.8 \%$ at day 0 vs $0.5 \%$ at day 120$)$ in the patient in parallel with substantial increase in the bacterial, fungal diversity and the abundance of Caudovirales bacteriophages.

In addition, Serial FMTs enhanced the ecological network of bacteria-fungi interactions in the recipient with a significant increase in these inter-kingdom correlations after each FMT. Conclusions We show that bacterial, fungal and virus communities respond differently to FMT. In addition to bacteria, future FMT practice should account for the significance of reconstituting gut fungi and viruses.

\section{IDDF2019-ABS-0161 APPLICATION OF FENTANYL COMBINED WITH MIDAZOLAM IN DIAGNOSIS AND TREATMENT OF LONGITUDINAL ENDOSCOPIC ULTRASONOGRAPHY AND OBSERVATION OF SEDATIVE EFFECT}

Rong Pei* ${ }^{*}$ Xiujiang Yang, Wenming Zhang, Yi Chen, Ke Chen. Shanghai cancer center hospital, China

10.1136/gutjnl-2019-IDDFabstracts. 173
Background To investigate the efficacy and safety of fentanyl combined with midazolam in the diagnosis and treatment of longitudinal endoscopic ultrasonography.

Methods A total of 260 patients undergoing longitudinal endoscopic ultrasonography were selected and randomly divided into observation group and control group with 130 cases in each group. Anxiety and fear patients were given psychological intervention before the operation. The observation group was anesthetized with fentanyl combined with midazolam by intravenous injection, while the control group was given $0.9 \%$ sodium chloride solution of equal volume. Heart rate and blood oxygen saturation were observed and compared before, during, at the end of the examination and 20 minutes after the operation. The total time of diagnosis and treatment, sedation depth and adverse reactions were observed and compared between the two groups.

Results HR in the observation group was lower than that before the examination, and HR in the control group was higher than that before the examination. Compared with the observation group at the same time, HR in the control group was higher during and at the end of the examination and the difference was statistically significant $(\mathrm{P}<$ $0.05)$. The incidence of side effects such as nausea, vomiting, cough, physical movement, mucosal hemorrhage, midway termination and operation time of the patients in the observation group were significantly lower than those in the control group, and the sedation effect, patient comfort and satisfaction of the patients were significantly higher than those in the control group, with statistical significance $(\mathrm{P}<0.05)$.

Conclusions Fentanyl combined with midazolam has a good sedative and analgesic effect in the diagnosis and treatment of longitudinal endoscopic ultrasonography and is safe.

\section{IDDF2019-ABS-0162 SARCOPENIA PREDICTS THE NEED FOR SURGERY AND CORRELATES WITH POSTOPERATIVE COMPLICATIONS IN SEVERE ULCERATIVE COLITIS PATIENTS}

Xiaolong Ge*, Wei Zhou. Sir Run Run Shaw Hospital, School of Medicine, Zhejiang University, China

\subsection{6/gutjnl-2019-IDDFabstracts. 174}

Background Acute severe ulcerative colitis (ASUC) is always known as a life-threatening condition which requires medical treatment including first-line treatment (intravenous corticosteroids) and rescue treatment (ciclosporin or infliximab) or surgical intervention. Studies demonstrate the prediction of sarcopenia in disease activity of Crohn's disease, but the prediction value for surgery and postoperative complications in ASUC remains unclear.

Methods This study included 168 patients with ASUC from our center. Skeletal muscle area (SMA), visceral fat area (VFA), and subcutaneous fat area (SFA) were assessed using abdominal CT scan by radiologists blinded to the outcome. The definition of sarcopenia was according to a skeletal muscle index of $2 / \mathrm{m}^{2}$ for men and $2 / \mathrm{m}^{2}$ for women. Whether sarcopenia predicted the need for surgery and postoperative complications or not was determined. 